\title{
Modulation of atypical brain activation during executive functioning in autism: a pharmacological MRI study of tianeptine
}

Robert H. Wichers ${ }^{1,2^{*}}$, James L. Findon ${ }^{1}$, Auke Jelsma ${ }^{1,3}$, Vincent Giampietro ${ }^{4}$, Vladimira Stoencheva ${ }^{2}$, Dene M. Robertson ${ }^{1,2}$, Clodagh M. Murphy ${ }^{1,2}$, Sarah Blainey ${ }^{1}$, Grainne McAlonan ${ }^{1,2}$, Christine Ecker ${ }^{5}$, Katya Rubia ${ }^{6}$, Declan G. M. Murphy ${ }^{1,2}$ and Eileen M. Daly ${ }^{1}$

\begin{abstract}
Background: Autism spectrum disorder (ASD) is associated with deficits in executive functioning (EF), and these have been suggested to contribute to core as well as co-occurring psychiatric symptoms. The biological basis of these deficits is unknown but may include the serotonergic system, which is involved both in regulating EF in neurotypical populations and in the pathophysiology of ASD. We previously demonstrated that reducing serotonin by acute tryptophan depletion (ATD) shifts differences in brain function during performance of EF tasks towards control levels. However, ATD cannot be easily used in the clinic, and we therefore need to adopt alternative approaches to challenge the serotonin system. Hence, we investigated the role of the serotonergic modulator tianeptine on EF networks in ASD.

Method: We conducted a pharmacological magnetic resonance imaging study, using a randomized double-blind crossover design, to compare the effect of an acute dosage of $12.5 \mathrm{mg}$ tianeptine and placebo on brain activation during two EF tasks (of response inhibition and sustained attention) in 38 adult males: 19 with ASD and 19 matched controls.

Results: Under placebo, compared to controls, individuals with ASD had atypical brain activation in response inhibition regions including the inferior frontal cortex, premotor regions and cerebellum. During sustained attention, individuals with ASD had decreased brain activation in the right middle temporal cortex, right cuneus and left precuneus. Most of the case-control differences in brain function observed under placebo conditions were abolished by tianeptine administration. Also, within ASD individuals, brain functional differences were shifted significantly towards control levels during response inhibition in the inferior frontal and premotor cortices.
\end{abstract}

Limitations: We conducted a pilot study using a single dose of tianeptine, and therefore, we cannot comment on long-term outcome.

Conclusions: Our findings provide the first evidence that tianeptine can shift atypical brain activation during EF in adults with ASD towards control levels. Future studies should investigate whether this shift in the biology of ASD is maintained after prolonged treatment with tianeptine and whether it improves clinical symptoms.

\footnotetext{
*Correspondence: rob.wichers@kcl.ac.uk

${ }^{1}$ Department of Forensic and Neurodevelopmental Sciences, The Sackler Centre for Translational Neurodevelopment, Institute of Psychiatry,

Psychology and Neuroscience, King's College London, PO50 De Crespigny Park, Denmark Hill, London SE5 8AF, UK

Full list of author information is available at the end of the article
}

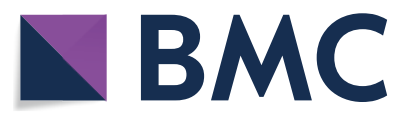

(c) The Author(s) 2021. Open Access This article is licensed under a Creative Commons Attribution 4.0 International License, which permits use, sharing, adaptation, distribution and reproduction in any medium or format, as long as you give appropriate credit to the original author(s) and the source, provide a link to the Creative Commons licence, and indicate if changes were made. The images or other third party material in this article are included in the article's Creative Commons licence, unless indicated otherwise in a credit line to the material. If material is not included in the article's Creative Commons licence and your intended use is not permitted by statutory regulation or exceeds the permitted use, you will need to obtain permission directly from the copyright holder. To view a copy of this licence, visit http://creativecommons.org/licenses/by/4.0/. The Creative Commons Public Domain Dedication waiver (http://creativeco mmons.org/publicdomain/zero/1.0/) applies to the data made available in this article, unless otherwise stated in a credit line to the data. 
Keywords: Autism spectrum disorder, Executive functioning, Tianeptine, Serotonin, fMRI

\section{Introduction}

Autism spectrum disorder (ASD) is a complex, heterogeneous, neurodevelopmental condition with an estimated population prevalence of 1:68 [1]. The cognitive phenotype of ASD includes atypical executive functioning (EF) [2,3], which comprises a range of cognitive processes that are necessary for concentrating and paying and/or switching attention [4]. It has been suggested that core ASD symptoms may contribute to alterations in EF, including response inhibition and sustained attention. For example, restricted, stereotyped and repetitive behaviours (RSRBs) have been associated with abnormalities in inhibitory control [5], which have frequently been reported in ASD [6]. Abnormalities in sustained attention networks are also thought to at least partially underpin both core (e.g. communication) [7] and associated (e.g. attention deficit hyperactivity disorder (ADHD)) ASD symptoms [7]. Targeting EF networks may therefore be of clinical value in treating core and associated symptoms in ASD.

Brain regions that are activated during EF tasks have been implicated in ASD. For example, functional differences in the inferior and orbitofrontal cortex, caudate, thalamus and cerebellum have been reported in children and adults with ASD as compared to typically developing control subjects during response inhibition tasks [8-10], which has been further confirmed in a recent meta-analysis of functional MRI (fMRI) studies of cognitive control [11]. Also, fMRI studies using sustained attention tasks have reported significantly less activation in children and adults with ASD [7, 12] in regions associated with sustained attention, including the inferior and middle frontal, parietal, striato-thalamic and cerebellar regions [13, 14]. The biological basis of these differences is unknown, but the serotonergic system may be involved. For example, in neurotypical populations, increasing brain serotonin levels with a selective serotonin reuptake inhibitor (SSRI) [15] has been shown to improve performance on a response inhibition task, but also to impair sustained attention [16] and to reduce brain activation in frontal and subcortical attention networks during a vigilance task [17].

The serotonergic system has also been implicated in the pathophysiology of ASD by prior genetic, biochemical and neuroimaging studies. For example, SLC6A4 (the serotonin transporter gene) has been linked to the diagnosis of ASD [18] and hyperserotonemia has been observed in approximately $30 \%$ of ASD individuals [19]. Neuroimaging studies have reported a significant reduction in cortical 5-HT2A receptor density [20] and in the binding of the serotonin transporter in adults with ASD [21]. In addition, more recent evidence for the role of serotonin in modulating EF in ASD includes a report that abnormal brain activation during performance of a Go/No-Go task was shifted significantly towards control levels after reducing serotonin by acute tryptophan depletion (ATD) [9]. Moreover, the degree of change in brain activation correlated with the severity of RSRBs, suggesting a potential treatment application. However, ATD is an experimental procedure that cannot easily be used in a routine clinical setting. Thus, repurposing a drug that reduces serotonin may provide a novel treatment opportunity that could be (relatively) quickly 'translated' to the clinic.

Tianeptine has been reported to, among other actions, enhance the reuptake of serotonin [22, 23] and to have cognitive enhancing abilities $[24,25]$. Hence, we tested the impact of tianeptine on brain function during an inhibitory and sustained attention task in ASD. Based on the study of ATD in adults with ASD [9] and tianeptine's effect on cognitive functions, we hypothesized that abnormalities in brain activation during a Go/No-Go task of response inhibition and a sustained attention task, as measured by functional MRI, would be abolished in the ASD group after a single dose of tianeptine. We further hypothesized that the degree of responsivity would be related to severity of core or associated symptoms. Therefore, we aimed to provide proof of concept that a single dose of tianeptine can shift atypical brain activation in ASD towards a more typical profile.

\section{Materials and methods \\ Participants}

Nineteen male, right-handed adults with ASD and 19 typically developed (TD) control participants were included in the study (age: ASD mean $=30, \mathrm{SD}=11$, TD mean $=27, \mathrm{SD}=9$ ). Two ASD cases and 2 TD controls were excluded from the Go/No-Go task due to significant head movement, leaving a sample of 17 ASD cases and 17 TD controls for the Go/No-Go task, and 19 ASD cases and 19 TD controls for the sustained attention task. The sample size was chosen based on results from our prior experiments targeting serotonin modulation using acute tryptophan depletion $[9,26]$, which were successful in detecting group differences in BOLD response with sample sizes of $n=14$. This implies an effect size (expressed in Cohen's d) in excess of $1.2[9,26]$. Exclusion criteria included medical disorders that could influence cognitive 
performance, major mental illnesses other than ASD, genetic disorders associated with ASD, alcohol or substance dependence or taking any medication affecting the serotonergic system (e.g. antidepressants, antipsychotics, benzodiazepines or mood stabilizers). The ASD diagnoses were made by a consultant psychiatrists using ICD10 research criteria [27] and confirmed using the Autism Diagnostic Interview-Revised (ADI-R) [28] if an informant was available. Current autistic symptoms were measured by the Autism Diagnostic Observation Schedule (ADOS) [29]. Intelligence was measured by the Wechsler Abbreviated Scale of Intelligence test (WASI) [30]. All participants completed baseline self-reported questionnaires of autistic traits (Autism-Spectrum Quotient) [31], obsessionality (Obsessive-Compulsive InventoryRevised) [32] and current symptoms of ADHD (Barkley Adult ADHD Rating Scale-IV) [33]. Symptoms of anxiety and depression were assessed using The Hamilton Rating Scales for Depression [34] and Anxiety [35]. All participants gave written, informed consent after receiving a complete description of the study. The study had National Research Ethics approval following review by the Stanmore Ethics Committee, London, UK.

\section{Tianeptine administration procedure}

Participants were required to complete two scanning sessions: one after receiving a single dose of $12.5 \mathrm{mg}$ of encapsulated tianeptine and one after receiving a dose of encapsulated placebo (ascorbic acid), in a randomized, double-blind, crossover design. A list of blinding numbers were produced independently and passed directly to the pharmacy in the outpatient department of the Maudsley Hospital, South London \& Maudsley NHS Trust, London, UK, using a computerized random number generator with blocked randomization. The pharmacy used these numbers to blind each dose (placebo; tianeptine) as they were encapsulated. Both subject and researcher(s) were blind to dosing throughout data acquisition. The randomization and encapsulation were conducted according to Good Medical Practice and in accordance with CONSORT and SPIRIT guidelines. Each dose was given to the participant $1 \mathrm{~h}$ prior to scanning, as tianeptine reaches its peak plasma level after approximately $1 \mathrm{~h}$ [36]. There was a minimum of eight days between the scans to allow for complete washout of the drug $\left(t^{1} / 2=3 \mathrm{~h}\right.$; washout $\left.=5^{*} \mathrm{t}^{1 / 2}=15 \mathrm{~h}\right)$. All participants received a screening by a medical doctor before and after the administration of both doses.

\section{Visual analogue scale}

All participants completed self-report visual analogue scale (VAS) questionnaires prior to drug administration and after the MRI scan. Side effects potentially associated with tianeptine were measured, including palpitations, nausea, dizziness, attentiveness, anxiety and irritability.

\section{Go/No-Go inhibition fMRI task}

In order to probe the brain's response inhibition system, participants engaged in a Go/No-Go task (GNG) during each scanning session $[8,37]$. During this task, participants made either a motor response on a button box to Go signals or inhibited this response to No-Go signals. In this task, arrows appear pointing to either the left or right side of the screen. The participant responds by pressing the left or right button as fast as possible on a diamond-shaped keypad. Infrequently (12\%), arrows pointing to the top (No-Go signals) appear. Subjects have to inhibit any motor response to these stimuli. In $12 \%$ of trials, slightly slanted (45 degrees) arrows pointing left or right (oddballs) appear and subjects have to respond as fast as they can, in the same way as for Go signals. No-Go responses were compared to successful oddball trials [8, 37]. There are two reasons we used the oddball instead of Go trials for the comparison. Firstly, this was done in order to control for the oddball effect of the No-Go trials. The No-Go trials are different from the Go trials and appear with less frequency, eliciting the so-called oddball attention effect. Participants pay more attention to rare stimuli than to high-frequent stimuli. Hence, the No-Go trials in addition to measuring inhibition also measure attention allocation to oddball stimuli. Furthermore, in order to control for this effect we added the oddball stimuli and contrasted No-Go with these oddball trials. Secondly, the Go trials appear with higher frequency than the No-Go trials. Hence, the oddball trials furthermore allow us to compare the same amount of No-Go vs oddball ('Go') stimuli.

\section{Sustained attention fMRI task}

In order to probe the brain's sustained attention network system, the Sustained Attention task (SAT) was performed during each scanning session [7, 12, 13]. In this task, participants need to respond via a right-hand button response as quickly as possible (i.e. within $1 \mathrm{~s}$ ) to the appearance of a visual timer counting up in milliseconds. When they press the button, the counter shows their reaction time in milliseconds. The visual stimuli appear either after short, predictable consecutive delays of $0.5 \mathrm{~s}$ (260 stimuli in total), in series of 3-5 consecutive stimuli or after unpredictable time delays of 2,5 or $8 \mathrm{~s}$ (20 each), which are pseudo-randomly interspersed into the blocks of 3-5 delays of $0.5 \mathrm{~s}$. The long, infrequent, unpredictable delays place a higher load on sustained attention, as participants have to wait for them to occur and they do not know the exact time when they will occur $(2 \mathrm{~s}$, $5 \mathrm{~s}$ or $8 \mathrm{~s}$ )-whereas the short, predictable 0.5-s delays 
appearing in a row are typically anticipated. Participants learn to estimate the $0.5 \mathrm{~s}$ and know that there will be several stimuli appearing in a row [38], placing a higher demand on sensorimotor synchronization [12].

We have previously consistently shown with this task that sustained attention networks are activated during the long relative to the short delays with progressively increasing activation in these networks from 2 to $8 \mathrm{~s}$ [7, $12,13]$. Here, we only report on the longest delay that elicits the strongest sustained attention activation, i.e. 8-s vs 0.5 -s delays.

\section{Baseline characteristics and task performance statistical analyses}

Statistical tests were performed using the SPSS software (v23.0) [39]. T-tests were used to compare baseline characteristics between groups and multivariate analysis of variance (MANOVA) determined any differences in performance and visual analogue scale outcome measures between group and drug conditions. Analysis of variance (ANOVA) was used to compare the largest displacement in head movement between group and drug conditions.

For the GNG task, the performance measures included: probability of inhibition (main inhibitory measure), mean reaction time to the Go signal (motor execution measure) and mean reaction time to the oddball signal. For the SAT task, the performance measures included: coefficient of variation (variation in reaction time during performance of the task adjusted for reaction time, i.e. standard deviation of reaction time divided by reaction time), mean reaction time, premature responses and omission errors.

\section{fMRI image acquisition}

All participants were scanned at the Centre for Neuroimaging Sciences, King's College London, on a 3-T General Electric Signa HD $\times$ Twinspeed scanner (Milwaukee, Wisc.), fitted with a quadrature birdcage head coil. For the fMRI, we acquired T2*-weighted volumes $(\mathrm{GNG}=260 ; \mathrm{SAT}=480)$ on non-adjacent slices $(\mathrm{GNG}=37 ; \mathrm{SAT}=31)$ parallel to the anterior-posterior commissure. For GNG, imaging parameters were: $\mathrm{TE}=30 \mathrm{~ms}, \mathrm{TR}=1.8 \mathrm{~s}$, flip angle $=73^{\circ}$, slice thickness $=3.0 \mathrm{~mm}$, in-plane voxel size $=3.75 \mathrm{~mm}^{2}$, slice gap $=0.7 \mathrm{~mm}$ and matrix size $=64 \times 64$ voxels. For SAT they were: $\mathrm{TE}=30 \mathrm{~ms}$, $\mathrm{TR}=1.5 \mathrm{~s}$, flip angle $=68^{\circ}$, slice thickness $=3.0 \mathrm{~mm}$, in-plane voxel size $=3.75 \mathrm{~mm}^{2}$, slice gap $=1.4 \mathrm{~mm}$, and matrix size $=64 \times 64$ voxels.

Also, a high-resolution gradient echo structural scan was sagittally acquired to be used during normalization of the fMRI data into Talairach space. Imaging parameters were: $\mathrm{TE}=30 \mathrm{~ms}, \mathrm{TR}=3 \mathrm{~s}$, flip angle $=90^{\circ}, 43$ slices, slice gap $=0.3 \mathrm{~mm}$, slice thickness $=3.0 \mathrm{~mm}$, matrix size $=128 \times 128$ voxels.

\section{fMRI image analysis}

The fMRI data were analysed using the XBAM (version 4) software developed at the King's College London's Institute of Psychiatry, Psychology and Neuroscience [40]. The associated methods are described in brief in this section and in more detail in Additional file 1. This nonparametric approach minimizes assumptions involved in image processing and has been previously described [26]. Within each run, every volume was realigned to the mean of all the images in the run and then smoothed (in native space) using a Gaussian filter (fullwidth at half-maximum $8.8 \mathrm{~mm}$ ). Using a wavelet-based resampling method, a time-series analysis was conducted on each individual subject, in order to compute a sum of squares (SSQ) ratio reflecting the BOLD effect. SSQ ratio maps were transformed into standard stereotactic space [41] using a two-stage warping procedure [40]. First, an average image intensity map for each individual was computed and then warped onto their structural scan. A second stage process then transformed each of these maps from structural space to Talairach space by maximizing the correlation between the images at each stage. The SSQ ratio maps were then transformed into Talairach space using these same two transformations. Group brain activation maps were computed for each drug condition with hypothesis testing performed at both the voxel and the cluster level. Using data-driven, permutation-based methods, with minimal distributional assumptions, time-series analyses were performed for group maps and inter-group random permutation for within-/between-group ANOVAs to compute the distribution of the SSQ ratio under the relevant null distribution hypothesis. Thresholding to the required level of significance was then performed using a two-stage process: first at a voxel-wise $p$-value of 0.05 , followed by grouping the supra-threshold voxels into 3D clusters and testing their significance against a null distribution of clusters occurring by chance in the permuted data. The cluster-wise $p$-value can thus be set in such a way as to yield less than one false-positive 3D cluster per map. For GNG, brain activations during No-Go responses were compared to brain activations during successful oddball trials. For SAT, brain activations during 8-s delays were compared to brain activations during 0.5-s delays. A group brain activation map was produced for each group (TD, ASD) and medication (placebo, tianeptine) status. Finally, all ANOVA analyses were conducted with voxel level $p<0.05$ and a cluster level $p<0.02$ determined as described above.

\section{Between-group analysis of variance}

A main effect of group (ASD, TD) analysis was conducted for the placebo condition for both GNG and SAT. 
To investigate whether brain activation differences in the ASD group relative to the control group under placebo changed after tianeptine dose in ASD, a main effect of group analysis was conducted in regions showing a main effect of group under placebo, but now comparing the control group on placebo with the ASD group on tianeptine, to test whether tianeptine would abolish the baseline differences.

Furthermore, a within ASD effect of drug analysis was conducted, in regions showing a main effect of group, to investigate whether the degree of change in activation in ASD following tianeptine was significant.

\section{Group $x$ drug status interaction analysis of variance}

A two-group (ASD, TD) by two-drug status (placebo, tianeptine) factorial repeated-measures ANOVA was conducted for each task. This analysis investigates how the BOLD response changes in brain regions in each group depending on drug status. The cluster-level threshold was adjusted to $p<0.02$, resulting in less than one false-positive cluster per map.

\section{Correlations between symptomatology and change in functional activations}

Pearson's correlations were conducted in XBAM to investigate any associations between core symptoms (as measured by the ADI-R and ADOS, 5 symptoms in total) and differences in BOLD response between tianeptine and placebo conditions (tianeptine-placebo) within ASD, in regions showing a main effect of group during placebo, during both tasks (8 regions in total). The SSQ ratio was extracted for each cluster showing a correlation and plotted versus symptomatology. A false-discovery-rate analysis was conducted to account and correct for multiple comparisons $(5 * 8=40$ comparisons in total).

\section{Results \\ Baseline characteristics}

The groups did not significantly differ in age and IQ. As expected, control subjects scored significantly lower on baseline autistic traits and symptoms of anxiety, obsessionality, depression, inattention (childhood) and hyperactivity (currently and in childhood). There was no significant difference between groups in current inattention scores (see Table 1).

\section{Visual analogue scales}

Despite baseline group differences in associated symptomatology (see Additional file 1: Table 1), multivariate analysis of variance showed no significant difference after placebo or tianeptine intake in both groups on subjective reports of physical and psychological side effect symptoms including palpitations, nausea, dizziness,

Table 1 Subjects characteristics

\begin{tabular}{|c|c|c|c|}
\hline & $\operatorname{ASD}(n=19)$ & $\mathrm{TD}(n=19)$ & $t$-test $p$ value \\
\hline Age & $30 \pm 11(19-50)$ & $27 \pm 9(19-52)$ & 0.3 \\
\hline $\mathrm{IQ}$ & $113 \pm 14(79-139)$ & $115 \pm 10(88-130)$ & 0.7 \\
\hline ADI-R_Communication & $17 \pm 9$ & - & \\
\hline ADI-R—Social Interaction & $14 \pm 8$ & - & \\
\hline ADI-R_-Repetitive Behaviour & $5 \pm 2$ & - & \\
\hline ADOS_Communication & $3 \pm 2$ & - & \\
\hline ADOS_Social Interaction & $6 \pm 2$ & - & \\
\hline $\mathrm{AQ}$ & $31 \pm 11$ & $12 \pm 7$ & $<0.001^{* * *}$ \\
\hline HAM-D & $6 \pm 4$ & $2 \pm 3$ & $0.001^{* *}$ \\
\hline HAM-A & $8 \pm 6$ & $3 \pm 4$ & $0.003^{* *}$ \\
\hline $\mathrm{OCl}-\mathrm{R}$ & $23 \pm 13$ & $8 \pm 9$ & $<0.001^{* * *}$ \\
\hline GAD-7 & $7 \pm 5$ & $3 \pm 3$ & $0.01^{*}$ \\
\hline Barkley Inattention Childhood Self & $3.3 \pm 3.0$ & $0.7 \pm 1.2$ & $0.002^{* *}$ \\
\hline Barkley Hyperactivity Childhood Self & $3.7 \pm 2.9$ & $1.1 \pm 1.9$ & $0.004^{* *}$ \\
\hline Barkley Inattention Currently Self & $1.4 \pm 1.8$ & $0.7 \pm 1.5$ & 0.2 \\
\hline Barkley Hyperactivity Currently Self & $1.2 \pm 1.3$ & $0.4 \pm 0.8$ & $0.004^{* *}$ \\
\hline
\end{tabular}

Data in table are shown as mean \pm standard deviation (range) ( $n=$ number of participants). $n=19$ for sustained attention task and $n=17$ for Go/No-Go task, which did not significantly affect between-group differences in baseline characteristics or visual analogue scale measures

$T D$ typically developed controls, $A S D$ individuals with autism spectrum disorder, ADOS Autism Diagnostic Observation Scale, $A D I-R$ Autism Diagnostic InterviewRevised, AQ Autism Quotient, HAM-D Hamilton Depression Rating Scale, HAM-A Hamilton Anxiety Rating Scale, OCI-R Obsessive-Compulsive Inventory Revised, GAD-7 Generalized Anxiety Disorder Assessment

Between group $t$-test: ${ }^{*} p<0.05 ;{ }^{* *} p<0.01 ;{ }^{* * *} p<0.001$ 
attentiveness, anxiety, depression and irritability (see Additional file 1: Table 1).

\section{fMRI task performance Go/No-Go task}

Multivariate analysis of variance revealed no significant between-group or within-group differences for the probability of inhibition or mean reaction time to the Go or oddball stimuli (see Additional file 1: Table 2).

\section{Sustained attention task}

Multivariate analysis of variance revealed significant differences between ASD and TD during both placebo and tianeptine conditions: slower mean reaction time and higher intrasubject variability for the 0.5- and 8-s delays in ASD compared to controls. More omission errors in ASD compared to controls were observed during the placebo (but not tianeptine) condition for the 0.5-s delay. There were more premature responses in ASD compared to controls during both drug conditions for the 0.5-s delay. There were no significant within-group differences in performance outcome following tianeptine in both groups. When comparing control subjects during placebo with ASD cases during tianeptine for all performance measures, ASD cases performed significantly worse compared to controls for the 0.5-s delay, but there were no significant differences for the 8-s delay (see Additional file 1: Table 3).

\section{Movement}

\section{Go/No-Go task}

Analysis of variance revealed, for largest head displacement in 3-dimensional space, no significant effect of group $(F(1,64)=1.64 ; p=0.21)$, drug $(F(1,64)=1.39$; $p=0.24)$ or group $\mathrm{x}$ drug interaction $(F(1,64)=0.003$; $p=0.95$ ) (see Additional file 1: Table 4).

\section{Sustained attention task}

Analysis of variance revealed, for largest head displacement in 3-dimensional space, no significant effect of group $(F(3,72)=3.13 ; p=0.08)$, drug $(F(3,72)=2.00$; $p=0.16)$ or group $\mathrm{x}$ drug interaction $(F(3,72)=0.22$; $p=0.64$ ) (see Additional file 1: Table 4).

\section{Within-group brain activations \\ Go/No-Go task}

The group activation maps for each group and drug status revealed significant activation during successful inhibition (No Go > oddball) in inhibitory modulating regions including the inferior, medial, middle frontal and premotor cortex and cerebellum (see Additional file 1: Fig. 1 and Tables 5-8).

\section{Sustained attention task}

The group activation maps for each group and drug status revealed significant activation during sustained attention ( $8 \mathrm{~s}>0.5 \mathrm{~s}$ ) in the superior and middle frontal, superior and middle temporal, occipital and pre- and postcentral cortices and cerebellum (see Additional file 1: Fig. 2 and Tables 9-12).

\section{Between-group differences in brain activation during placebo and tianeptine Go/No-Go task}

During placebo, subjects with ASD relative to TD showed a decrease in BOLD signal in the right postcentral cortex $(p=0.009$, cluster size $=144$ voxels $)$. By contrast, increased activation in ASD compared to TD was observed in the left inferior frontal cortex/left insula ( $p=0.02$, cluster size $=95$ voxels $)$, right premotor cortex $(p=0.01$, cluster size $=111$ voxels $)$, right cerebellum $(p=0.02$, cluster size $=81$ voxels $)$ and right occipital cortex $(p=0.01$, cluster size $=114$ voxels) (see Fig. 1a and Table 2).

In order to test whether tianeptine would abolish the baseline differences, subjects with ASD after the tianeptine dose were compared to TD individuals after the placebo dose, focusing on regions where betweengroup differences under placebo were observed. Nearly all between-group differences were abolished, leaving only one small increase in activation in subjects with ASD compared to controls in the right lingual cortex $(p=0.007$, cluster size $=45$ voxels) (see Fig. $1 \mathrm{~b}$ and Table 2). Subsequently, a within-ASD analysis was conducted to investigate the effect of drug in those regions specifically. A significant decrease in brain activation was observed in the left insula ( $p=0.04$, see Fig. 2$)$ and right precentral cortex ( $p=0.01$, see Fig. 2).

\section{Sustained attention task}

During placebo, subjects with ASD relative to TD showed decreased BOLD signal in the right middle temporal cortex $(p=0.02$, cluster size $=212$ voxels $)$, right cuneus ( $p=0.001$, cluster size $=1082$ voxels $)$ and left precuneus $(p=0.009$, cluster size $=747$ voxels $)$ (see Fig. $1 c$, Table 2$)$.

In order to test whether tianeptine would abolish the baseline differences, subjects with ASD during tianeptine were compared to TD during placebo focusing on regions where between-group differences under placebo were observed. Nearly all of these between-group differences were no longer observed, leaving only one small decrease in activation in subjects with ASD compared to TD in the right cuneus $(p=0.003$, cluster size $=385$ voxels) (see Fig. 1d and Table 2). Subsequently, a within-ASD analysis was conducted to investigate the effect of drug 
Table 2 Anatomical location and statistics for BOLD activation

\begin{tabular}{|c|c|c|c|c|c|}
\hline Region & $x$ & $Y$ & $Z$ & Cluster $p$ value & Cluster size \\
\hline \multicolumn{6}{|l|}{ GO/NO-GO task ( $n=17$ for both groups) } \\
\hline \multicolumn{6}{|l|}{ ASD placebo vs TD placebo } \\
\hline \multicolumn{6}{|l|}{$A S D<T D$ (blue) } \\
\hline Right postcentral cortex & 58 & -19 & 33 & 0.009 & 144 \\
\hline \multicolumn{6}{|l|}{$A S D>T D($ red $)$} \\
\hline Right cerebellum & 29 & -67 & -40 & 0.02 & 81 \\
\hline Right occipital cortex & 11 & -96 & -7 & 0.01 & 114 \\
\hline Left inferior frontal cortex/left insula & -40 & 19 & 13 & 0.02 & 95 \\
\hline Right premotor cortex & 43 & -7 & 50 & 0.01 & 111 \\
\hline \multicolumn{6}{|l|}{ ASD tianeptine vs TD placebo } \\
\hline \multicolumn{6}{|l|}{$A S D<T D$ (blue) } \\
\hline Right lingual cortex & 11 & -100 & -3 & 0.007 & 45 \\
\hline \multicolumn{6}{|c|}{ Interaction of drug status (placebo, tianeptine) by group (ASD, TD) } \\
\hline \multicolumn{6}{|l|}{ Cerebellum bilaterally/limbic area } \\
\hline Right rostromedial frontal & 25 & -19 & -20 & 0.008 & 357 \\
\hline Cortex/caudate/cingulate & 29 & 59 & 7 & 0.009 & 376 \\
\hline \multicolumn{6}{|c|}{ Sustained attention task ( $n=19$ for both groups) } \\
\hline \multicolumn{6}{|l|}{ TD placebo vs ASD placebo } \\
\hline \multicolumn{6}{|l|}{$A S D<T D$ (blue) } \\
\hline Right Middle Temporal Cortex & 61 & -7 & -7 & 0.02 & 212 \\
\hline Right cuneus & 14 & -93 & 3 & 0.0008 & 1082 \\
\hline Left precuneus & -4 & -63 & 46 & 0.009 & 747 \\
\hline \multicolumn{6}{|l|}{ TD placebo vs ASD tianeptine } \\
\hline \multicolumn{6}{|l|}{$A S D<T D$ (blue) } \\
\hline Right cuneus & 14 & -93 & 3 & 0.003 & 385 \\
\hline \multicolumn{6}{|c|}{ Interaction of drug status (placebo, tianeptine) by group (ASD, TD) } \\
\hline Right middle temporal cortex & 40 & -56 & 10 & 0.01 & 204 \\
\hline Right thalamus & 7 & -11 & 7 & 0.001 & 502 \\
\hline Left middle frontal cortex & -51 & 4 & 46 & 0.02 & 180 \\
\hline
\end{tabular}

$X, Y, Z=$ Peak Talairach coordinates

$B O L D$ blood-oxygen-level-dependent, $A S D$ Individuals with Autism Spectrum Disorder, TD Typically Developed Controls

in those regions specifically. In none of the regions, brain activation changed significantly.

\section{Group by drug interaction effects Go/No-Go task}

There were significant interaction effects of BOLD signal response between drug status (placebo, tianeptine) and group (ASD, TD) in two clusters including the right rostromedial frontal cortex (extending into anterior cingulate cortex and caudate; $p=0.008$, cluster size $=357$ voxels) and the cerebellum bilaterally (extending into parahippocampal cortex; $p=0.009$, cluster size $=376$ voxels). In the right rostromedial frontal cortex tianeptine decreased BOLD signal in the TD group, whereas it increased BOLD signal in ASD. The opposite was observed in the cerebellum (see Fig. 3a and Table 2).

\section{Sustained attention task}

There were significant interaction effects of BOLD signal response between drug status (placebo, tianeptine) and group (ASD, TD) in three clusters. These included the right middle temporal cortex $(p=0.01$, cluster size $=204$ voxels), right thalamus $(p=0.001$, cluster size $=502$ voxels) and left middle frontal cortex ( $p=0.02$, cluster size $=180$ voxels). In the right middle temporal cortex tianeptine increased BOLD signal in the TD group, whereas it decreased BOLD signal in ASD. The opposite pattern was observed in the right thalamus and left middle frontal cortex where tianeptine decreased activation in the TD group and increased it in ASD (see Fig. 3b and Table 2). 


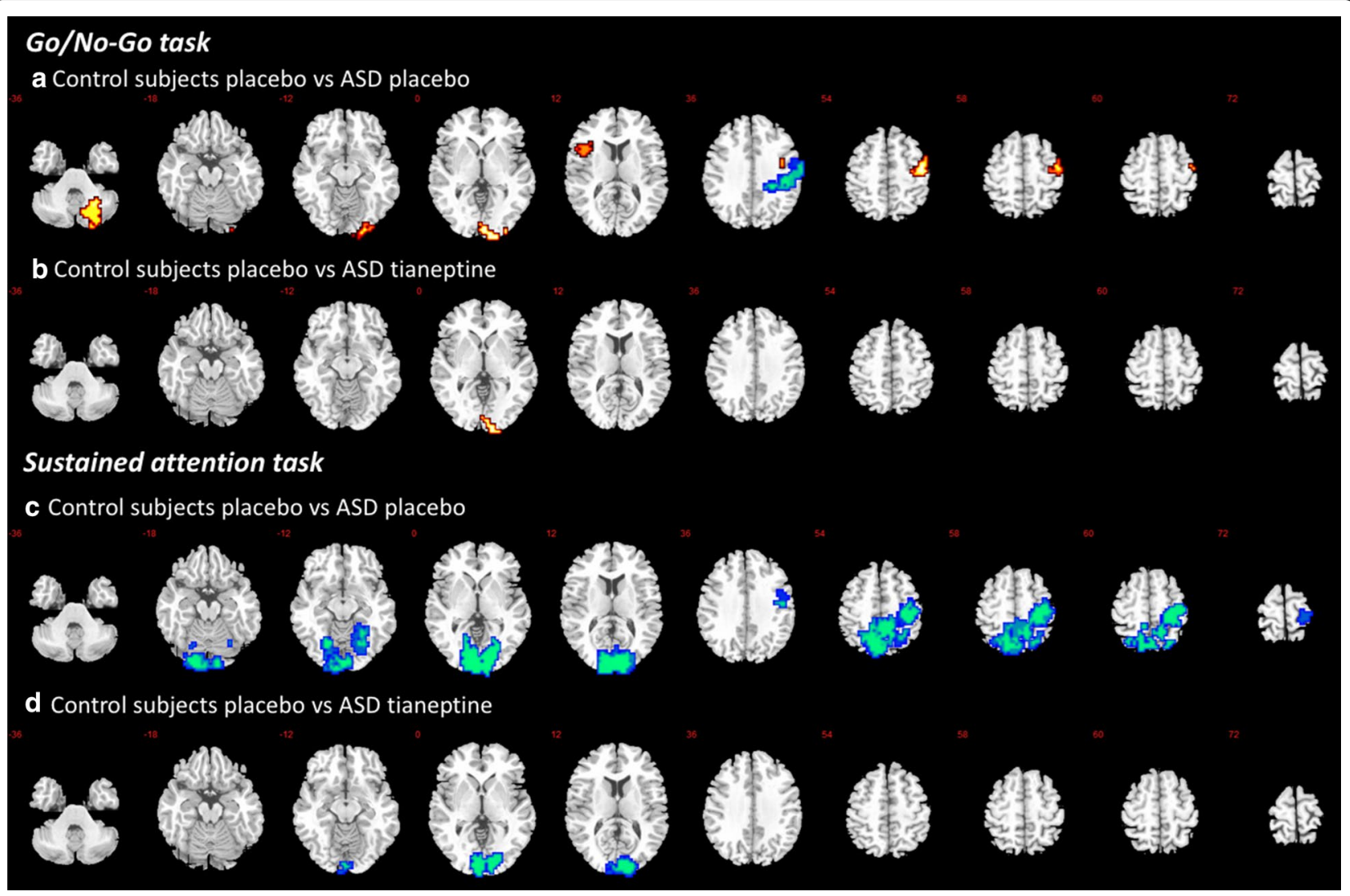

Fig. 1 Brain activation map showing abnormally activated regions during response inhibition and sustained attention in ASD that were no longer observed following tianeptine administration; $p<0.02$ at cluster level. Location of BOLD signal changes between groups. Red: ASD > TD; Blue: ASD $<$ TD. Numeric label $=z$ Talairach coordinate. Right hemisphere of brain is on the right side of the image. BOLD blood-oxygen-level-dependent, $A S D$ Individuals with autism spectrum disorder, TD typically developed controls

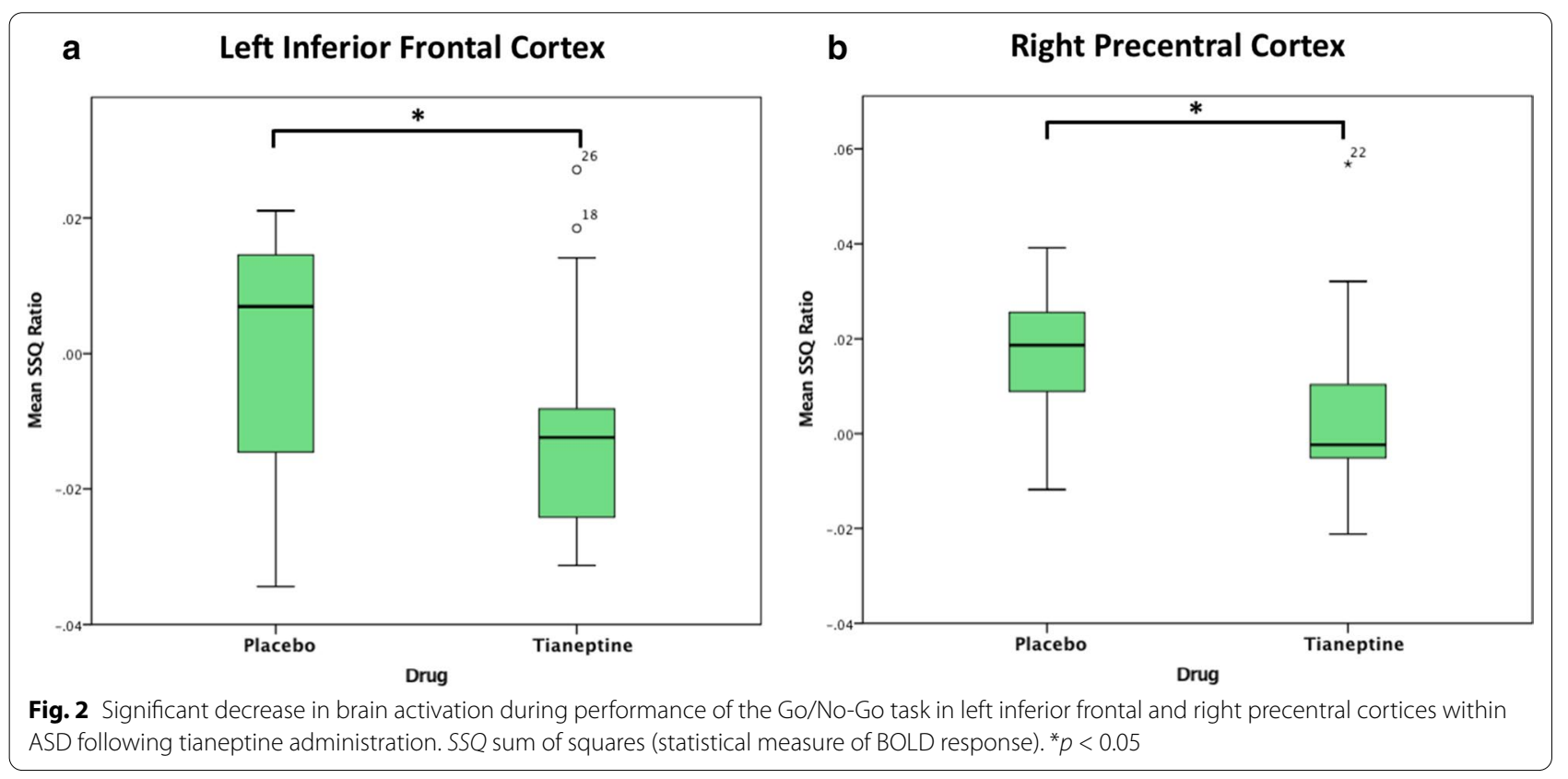




\section{a Go/No-Go task}

\section{Right Rostromedial Frontal Cortex}

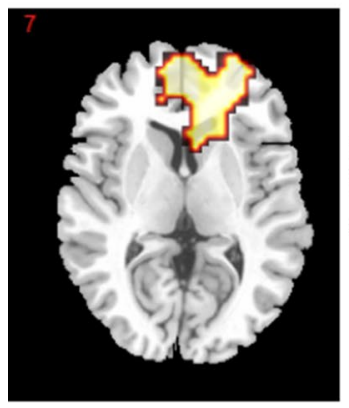

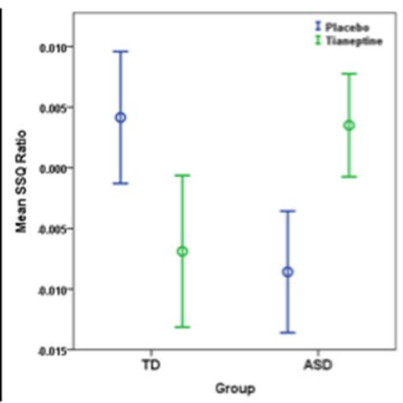

Group
Cerebellum
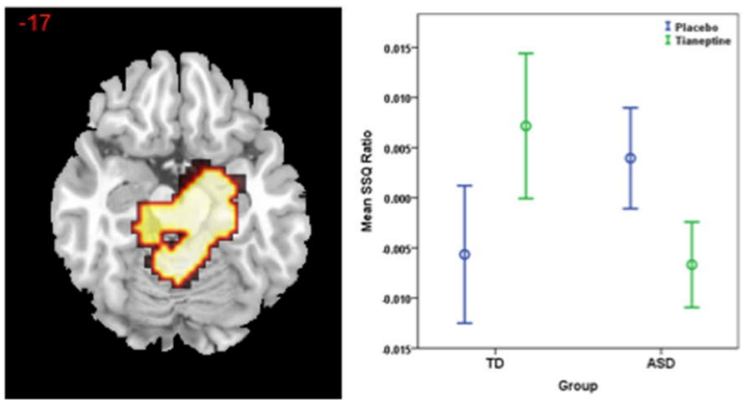

\section{b Sustained Attention task}

\section{Right Middle Temporal Cortex}

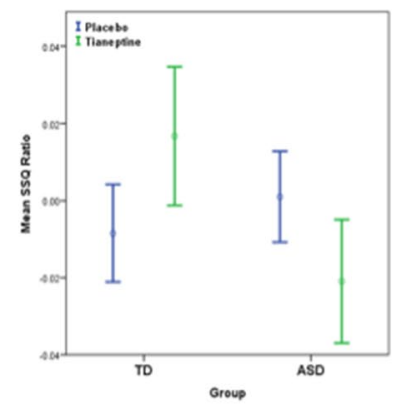

Right Thalamus

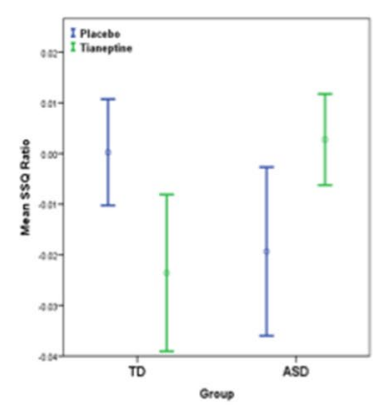

Left Middle Frontal Cortex

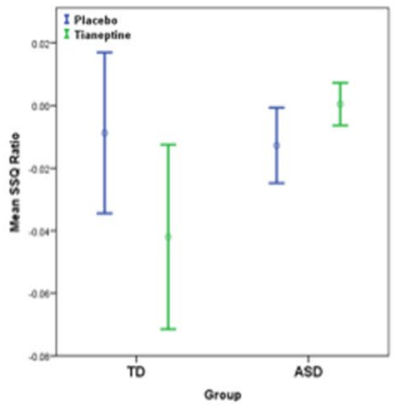

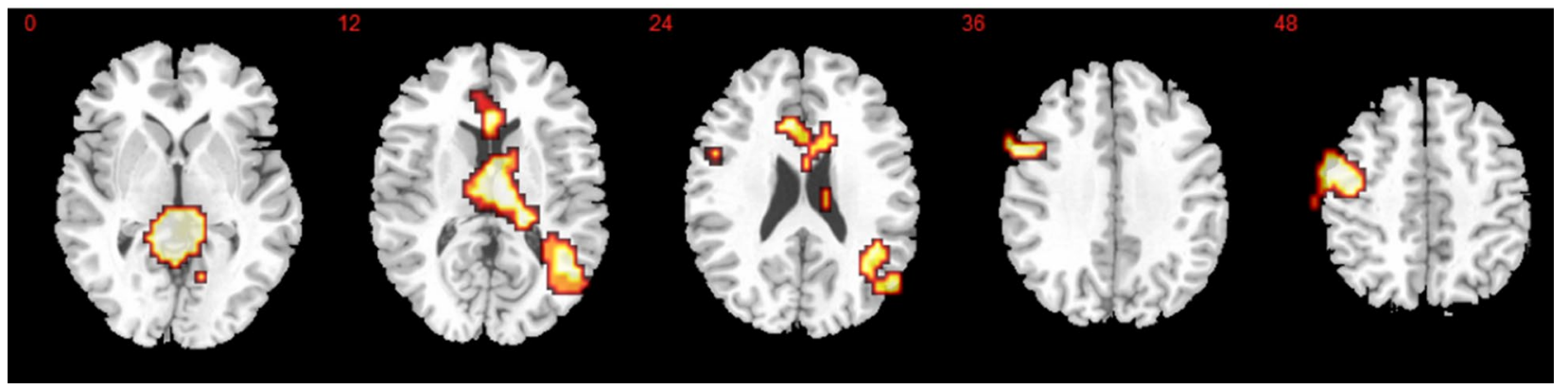

Fig. 3 Interaction of drug status (placebo, tianeptine) by group (ASD, TD) during EF; $p<0.02$ at cluster level. Location of BOLD signal for ANOVA interaction. Numeric label = z Talairach coordinate. Box plots: Mean BOLD signal extracted from each interaction cluster. Right hemisphere of brain is on the right side of the image. SSQ sum of squares FMRI statistic, BOLD blood-oxygen-level-dependent, ASD Individuals with autism spectrum disorder, TD Typically developed controls

Pearson's correlations between functional activations and symptomatology within ASD

\section{Go/No-Go task}

Within ASD, we observed correlations between change in brain activation following tianeptine and the severity of RSRBs. The degree of BOLD signal change between tianeptine and placebo correlated positively with severity of RSRBs in the right precentral cortex ( $r=0.90, p=<0.001$; at baseline ASD $<\mathrm{TD}$, extending from right postcentral cortex) and negatively in the right cerebellum $(r=-0.74, p=<0.02$, at baseline ASD $>$ TD). Hence, the more severe an individual's RSRB scores were at baseline, the more likely their BOLD signal in the right precentral cortex and cerebellum would shift towards control levels after tianeptine. 


\section{Sustained attention task}

Within ASD, we observed a correlation between brain activation and communication. Under placebo condition, there was a significant negative correlation between BOLD signal in the right cuneus and ADOS communication scores $(r=-0.59, p=0.01)$.

\section{Discussion}

To our knowledge, this is the first study to investigate the effect of tianeptine on brain activation (i.e. BOLD signal change) in adult males with ASD. We observed, under placebo condition, atypical brain activation in ASD during successful completion of the GNG, in the inferior frontal cortex, premotor regions and in the cerebellum. During completion of the SAT, we observed reduced brain activation in ASD compared to TD in the right middle temporal cortex, right cuneus and left precuneus. Furthermore, within the ASD group, the degree of atypical brain activation in the right cuneus was associated with ASD symptom severity (ADOS communication domain). Also, ASD cases performed significantly worse in SAT, as compared to TD. Following tianeptine exposure, within ASD, most brain-functional differences during both tasks were abolished and during response inhibition brain activation in the left inferior frontal and right premotor cortices shifted significantly towards control levels. Further work is required to investigate whether this shift of brain activation during EF tasks is maintained by prolonged tianeptine treatment.

Also, within individuals with ASD we observed correlations between the degree of change in BOLD signal (tianeptine-placebo) during response inhibition in the cerebellum and precentral cortex and the severity of RSRBs. These results are in line with a previous study of adult males with ASD that employed the same scanning paradigm, but decreased brain 5-HT with ATD [9]. This study also reported functional abnormalities in brain activation in the inferior frontal cortex and cerebellum. In addition, increased activation in the left inferior frontal cortex in ASD has previously been reported during motor response inhibition using the same GNG, during cognitive interference inhibition [8] and consistently in a meta-analysis of cognitive control fMRI studies [11]. Taken together, this provides preliminary evidence suggesting that tianeptine affects brain regions associated with RSRBs, though it is unknown if it can successfully treat RSRBs in ASD.

The findings of decreased brain activation during sustained attention in the right middle temporal cortex, right cuneus and left precuneus differ somewhat from previous fMRI studies using the same task in ASD. In these studies decreased brain activation was observed in prefrontal, parietal, temporal, striato-thalamic and cerebellar regions as well as a negative correlation with brain activation and age in the left precuneus and right occipital cortex $[7,12]$. The dissimilarity in findings may be explained by the different age ranges studied. One study only included children (ages: 11-17 years) with ASD [12], while the other study sample consisted of a mixture of children and adults (ages: 11-35 years) [7]. Nonetheless, our findings support the suggestion that individuals with ASD have abnormalities in brain activation during sustained attention-although the specific brain regions affected may be age-dependent.

The observed differences in task performance in ASD included slower mean reaction times and larger intrasubject response variability. This is consistent with what has been seen in previous studies in children and adults with ASD during attention tasks [7, 42]. Although tianeptine did not significantly change sustained attention performance in both groups, some performance abnormalities in ASD were no longer significant following tianeptine. This is in line with a study that reported improvement in neurocognitive functions in a neurotypical population after 12 weeks of treatment with tianeptine [25].

Further to the main effects of group, the interaction analysis results showed that, during response inhibition, within the control group, tianeptine decreased brain activation in the rostromedial frontal cortex and caudate, whereas in ASD brain activation was increased. In contrast, within the control group, tianeptine increased brain activation in the cerebellum, whereas it decreased cerebellum activation in ASD. This 'reversal' of brain activation may reflect altered functionality of frontal-cerebellar networks. Atypical connectivity of white matter within the cerebellum and its mid-brain and cortical projections have been observed in ASD [43, 44]. Studies investigating functional connectivity support these findings, suggesting abnormalities in connections between the cerebellum and both motor and non-motor cortical regions [45, 46]. Furthermore, the interactions found here are similar to previous reports of the impact of ATD on brain function in ASD compared to controls: the same direction of increases and decreases of brain activation was previously reported in the frontal cortex and cerebellum [9]. For the sustained attention task, the interaction analysis revealed three significant clusters where tianeptine shifted brain activation in opposite directions in ASD compared to controls. Within the control group, tianeptine increased brain activation in the right middle temporal cortex, whereas in ASD brain activation was decreased. In contrast, within the control group, tianeptine decreased brain activation in the right thalamus and left middle frontal cortex, whereas it increased brain activation in ASD. All these regions 
have previously been reported to either show abnormal brain activation and/or functional maturation in ASD during sustained attention [7]. In addition, our results are in line with prior imaging studies in ASD $[47,48]$. This suggests that brain regions implicated in the abnormal neurodevelopmental trajectory of ASD continue to show differences in adulthood, and this may have implications for treatment response. Taken together, our work and that of others suggest that the neuropharmacological mechanism underpinning response to tianeptine is different in ASD as compared to controls; therefore, treatments commonly used in neurotypical populations may not be as 'translatable' to individuals with ASD as currently assumed. Moreover, recently published treatment guidelines in ASD reported that evidence for the effectiveness of selective serotonin reuptake inhibitors (SSRIs) is limited [49]. Thus, pharmacological interventions affecting the serotonergic system need to be tested specifically in ASD as similar results to those found in neurotypical populations cannot be assumed.

Whether the impact of both ATD and tianeptine on abnormal brain activation in ASD is explained by the same neurochemical pathway-the serotonergic system-is unknown. Tianeptine was initially considered to be a selective serotonin reuptake enhancer (SSRE) as its acute and long-term administration decreased extracellular 5-HT levels in the brain stem, striatum, cerebral cortex and hippocampus in rats [22, 23]. Later studies, however, contradicted these findings-albeit this discrepancy may be explained by technical differences in the micro-dialysis techniques employed. Nevertheless, recent evidence in humans demonstrates a reduction in plasma serotonin and increase in platelet serotonin following acute administration of tianeptine, consistent with the effect of enhanced serotonin reuptake [50]. In addition, tianeptine has also been shown to: (1) regulate stress-induced glutamate release, (2) modulate plasticity in the amygdala; (3) reverse stress-induced hippocampal dystrophy [51]; and (4) be a $\mu$-opioid receptor agonist [52]. Given that we only investigated the brain response after a single dose of tianeptine, it is unlikely that our results are due to modulating plasticity or the reversal of dystrophy. However, we cannot rule out the possibility that our results may be partially explained by modulation of the glutamatergic and $\mu$-opioid systems. For example, the glutamatergic system has been widely reported to be abnormal in ASD [53]. Also, alterations in the $\mu$-opioid system have been proposed to contribute to ASD [54]. Hence, future studies are required to investigate which of the proposed mechanisms underlie the reported modulating effect.

\section{Limitations}

We conducted a pilot study using a single dose of tianeptine and so we cannot comment on long-term outcome. Nevertheless, this study does provide a first necessary proof of concept for a potential treatment targeting ASD symptomatology. Also, a potential problem with fMRI adaptations of response inhibition tasks is that motor responses to Go trials are compared to non-motor responses to No-Go trials. Thus, some activation differences could have been potentially motorrelated rather than purely inhibition-related. Last, we did not find differences in performance outcome between groups for the GNG. A recent study using a much larger sample (201 ASD cases and 240 controls) employed online GNG and reported deficits in response inhibition that were associated with diagnosis and autistic traits [6]. In contrast, lack of performance difference was reported in a similarly sized fMRI study using the GNG [9]. Hence, our sample size may be underpowered to detect behavioural data differences. However, the sample size was large enough to detect brain activation differences, which have previously been reported to be more sensitive to drug effects than behaviour, including in fMRI studies of ASD $[9,55]$.

\section{Conclusions}

We report that tianeptine can abolish most case-control differences in brain function during EF tasks; within ASD it can significantly shift brain activation deficits associated with RSRB towards control levels. This suggests a potential utility of tianeptine for targeting core or associated symptoms in ASD. Hence, future trials should investigate whether the shift in brain activation we discovered following a single dosage of tianeptine is maintained after prolonged treatment, and whether this is associated with response to treatment.

\section{Supplementary Information}

The online version contains supplementary material available at https://doi. org/10.1186/s13229-021-00422-0.

Additional file 1. Supplementary material.

\section{Abbreviations}

ASD: Autism spectrum disorder; EF: Executive functioning; RSRBs: Restricted, stereotyped and repetitive behaviours; ADHD: Attention deficit hyperactivity disorder; fMRI: Functional MRI; SSRI: Selective serotonin reuptake inhibitor; ATD: Acute tryptophan depletion; TD: Typically developed control participants; ADI-R: Autism Diagnostic Interview-Revised; ADOS: Autism Diagnostic Observation Schedule; WASI: Wechsler Abbreviated Scale of Intelligence test; VAS: Visual analogue scale; GNG: Go/No-Go task; SAT: Sustained attention task; MANOVA: Multivariate analysis of variance; ANOVA: Analysis of variance; SSQ: Sum of squares; SSRE: Selective serotonin reuptake enhancer. 


\section{Acknowledgements}

The authors would like to thank all the volunteers for their participation. The authors acknowledge support from the Clinical Biochemistry Department at King's College Hospital, the Maudsley Pharmacy Department and the Neurodevelopmental Clinic at the Maudsley Hospital. The authors acknowledge support from the National Institute for Health Research (NIHR) Biomedical Research Centre for Mental Health at South London and Maudsley NHS Foundation Trust and Institute of Psychiatry, Psychology and Neuroscience King's College London. This article presents independent research funded by the National Institute for Health Research (NIHR). The views expressed are those of the author(s) and not necessarily those of the NHS, the NIHR or the Department of Health.

\section{Authors' contributions}

RW collected, analysed and interpreted the data and was the major contributor in writing the manuscript. JF collected, analysed and interpreted the data. AJ analysed and interpreted the data. VG interpreted the data. VS, DM, CM, and SB collected the data. GM, CE, KR, DM and ED interpreted the data. DM and $E D$ were major contributors in writing the manuscript. All authors read and approved the final manuscript.

\section{Funding}

This study was funded by a grant (to Prof. D.G Murphy) from European Autism Interventions-A Multicentre Study for Developing New Medications (EUAIMS) (receiving support from the Innovative Medicines Initiative Joint Undertaking under Grant Agreement 115300, which includes financial contributions from the EU Seventh Framework Programme [FP7/2007-2013] and from the European Federation of Pharmaceutical Industries and Associations), a Grant from AIMS-2-TRIALS (to Prof. D.G. Murphy), a Grant (to Prof. D.G Murphy) from NIHR Mental Health Biomedical Research Centre Infrastructure Support for Pilot Studies and a Grant (to Prof. D.G Murphy) from the Sackler Institute for Translational Neurodevelopment.

\section{Availability of data and materials}

The dataset used and/or analysed during the current study are available from the corresponding author on reasonable request.

\section{Ethics approval and consent to participate}

All participants gave written, informed consent to participate in the study after receiving a complete description of the study. The study had National Research Ethics approval following review by the Stanmore Ethics Committee, London, UK (14/LO/0663).

\section{Consent for publication}

This manuscript does not contain any individual's data, so therefore this is not applicable.

\section{Competing interests}

Professor K. Rubia has received speaker's honoraria from Shire and Medicine and grants from Lilly and Shire. The other authors declare that they have no competing interests.

\footnotetext{
Author details

${ }^{1}$ Department of Forensic and Neurodevelopmental Sciences, The Sackler Centre for Translational Neurodevelopment, Institute of Psychiatry, Psychology and Neuroscience, King's College London, PO50 De Crespigny Park, Denmark Hill, London SE5 8AF, UK. ${ }^{2}$ Behavioural and Developmental Psychiatry Clinical Academic Group, South London and Maudsley NHS Trust, London, UK. ${ }^{3}$ VU University Medical Center, Amsterdam, The Netherlands. ${ }^{4}$ Department of Neuroimaging, Institute of Psychiatry, Psychology and Neuroscience, King's College London, London, UK. ${ }^{5}$ Department of Child and Adolescent Psychiatry, Psychosomatics and Psychotherapy, University Hospital Frankfurt am Main, Goethe-University, Frankfurt am Main, Germany. ${ }^{6}$ Department of Child and Adolescent Psychiatry, Institute of Psychiatry, Psychology and Neuroscience, King's College London, London, UK.
}

Received: 18 August 2020 Accepted: 8 February 2021

Published online: 19 February 2021

\section{References}

1. Christensen DL, Baio J, Van Naarden BK, Bilder D, Charles J, Constantino $\mathrm{JN}$, et al. Prevalence and characteristics of autism spectrum disorder among children aged 8 years-autism and developmental disabilities monitoring network, 11 sites, United States, 2012. MMWR Surveill Summ. 2016;65(3):1-23.

2. Hughes C, Russell J, Robbins TW. Evidence for executive dysfunction in autism. Neuropsychologia. 1994;32(4):477-92.

3. Rumsey JM, Hamburger SD. Neuropsychological findings in high-functioning men with infantile autism, residual state. J Clin Exp Neuropsychol. 1988;10(2):201-21.

4. Diamond A. Executive functions. Annu Rev Psychol. 2013;64:135-68.

5. Mosconi MW, Kay M, D'Cruz AM, Seidenfeld A, Guter S, Stanford LD, et al. Impaired inhibitory control is associated with higher-order repetitive behaviors in autism spectrum disorders. Psychol Med. 2009;39(9):1559-66.

6. Uzefovsky F, Allison C, Smith P, Baron-Cohen S. Brief report: the Go/ No-Go task online: inhibitory control deficits in autism in a large sample. J Autism Dev Disord. 2016;46(8):2774-9.

7. Murphy CM, Christakou A, Daly EM, Ecker C, Giampietro V, Brammer M, et al. Abnormal functional activation and maturation of fronto-striatotemporal and cerebellar regions during sustained attention in autism spectrum disorder. Am J Psychiat. 2014;171(10):1107-16.

8. Schmitz N, Rubia K, Daly E, Smith A, Williams S, Murphy DG. Neural correlates of executive function in autistic spectrum disorders. Biol Psychiat. 2006;59(1):7-16.

9. Daly E, Ecker C, Hallahan B, Deeley Q, Craig M, Murphy C, et al. Response inhibition and serotonin in autism: a functional MRI study using acute tryptophan depletion. Brain. 2014;137:2600-10.

10. Chantiluke K, Barrett N, Giampietro V, Santosh P, Brammer M, Simmons $\mathrm{A}$, et al. Inverse fluoxetine effects on inhibitory brain activation in non-comorbid boys with ADHD and with ASD. Psychopharmacology. 2015;232(12):2071-82.

11. Lukito S, Norman L, Carlisi C, Radua J, Hart H, Simonoff E, et al. Comparative meta-analyses of brain structural and functional abnormalities during cognitive control in attention-deficit/hyperactivity disorder and autism spectrum disorder. Psychol Med. 2020;50(6):894-919.

12. Christakou A, Murphy CM, Chantiluke K, Cubillo Al, Smith AB, Giampietro $\checkmark$, et al. Disorder-specific functional abnormalities during sustained attention in youth with Attention Deficit Hyperactivity Disorder (ADHD) and with autism. Mol Psychiatry. 2013;18(2):236-44.

13. Lim L, Hart H, Mehta MA, Simmons A, Mirza K, Rubia K. Neurofunctional abnormalities during sustained attention in severe childhood abuse. PLoS ONE. 2016;11(11):e0165547.

14. Lawrence NS, Ross TJ, Hoffmann R, Garavan H, Stein EA. Multiple neuronal networks mediate sustained attention. J Cogn Neurosci. 2003;15(7):1028-38.

15. Homberg JR. Serotonin and decision making processes. Neurosci Biobehav Rev. 2012;36(1):218-36

16. Riedel WJ, Eikmans K, Heldens A, Schmitt JA. Specific serotonergic reuptake inhibition impairs vigilance performance acutely and after subchronic treatment. J Psychopharmacol. 2005;19(1):12-20.

17. Wingen M, Kuypers KP, van de Ven V, Formisano E, Ramaekers JG. Sustained attention and serotonin: a pharmaco-fMRI study. Hum Psychopharmacol. 2008;23(3):221-30.

18. Yonan AL, Palmer AA, Smith KC, Feldman I, Lee HK, Yonan JM, et al. Bioinformatic analysis of autism positional candidate genes using biological databases and computational gene network prediction. Genes Brain Behav. 2003;2(5):303-20.

19. Gabriele S, Sacco R, Persico AM. Blood serotonin levels in autism spectrum disorder: a systematic review and meta-analysis. Eur Neuropsychopharmacol J Eur Coll Neuropsychopharmacol. 2014;24(6):919-29.

20. Murphy DG, Daly E, Schmitz N, Toal F, Murphy K, Curran S, et al. Cortical serotonin 5-HT2A receptor binding and social communication in adults with Asperger's syndrome: an in vivo SPECT study. Am J Psychiatry. 2006;163(5):934-6.

21. Nakamura K, Sekine Y, Ouchi Y, Tsujii M, Yoshikawa E, Futatsubashi M, et al. Brain serotonin and dopamine transporter bindings in adults with highfunctioning autism. Arch Gen Psychiatry. 2010;67(1):59-68.

22. Mennini T, Mocaer E, Garattini S. Tianeptine, a selective enhancer of serotonin uptake in rat-brain. N-S Arch Pharmacol. 1987:336(5):478-82. 
23. Fattaccini CM, Bolanosjimenez F, Gozlan H, Hamon M. Tianeptine stimulates uptake of 5-hydroxytryptamine invivo in the rat-brain. Neuropharmacology. 1990;29(1):1-8.

24. Klasik A, Krysta K, Krupka-Matuszczyk I. Effect of tianeptine on cognitive functions in patients with depressive disorders during a 3-month observation. Psychiatr Danub. 2011;23(Suppl 1):S18-22.

25. Jeon HJ, Woo JM, Lee SH, Kim EJ, Chung S, Ha JH, et al. Improvement in subjective and objective neurocognitive functions in patients with major depressive disorder: a 12-week, multicenter, randomized trial of tianeptine versus escitalopram, the CAMPION study. J Clin Psychopharmacol. 2014;34(2):218-25.

26. Daly EM, Deeley Q, Ecker C, Craig M, Hallahan B, Murphy C, et al. Serotonin and the neural processing of facial emotions in adults with autism: an fMRI study using acute tryptophan depletion. Arch Gen Psychiatry. 2012;69(10):1003-13.

27. WHO. The ICD-10 classification of mental and behavioural disorders: diagnostic criteria for research. Geneva: World Health Organizartion; 1993.

28. Lord C, Rutter M, Lecouteur A. Autism diagnostic interview-revised: a revised version of a diagnostic interview for caregivers of individuals with possible pervasive developmental disorders. J Autism Dev Disord. 1994;24(5):659-85.

29. Lord C, Rutter M, Goode S, Heemsbergen J, Jordan H, Mawhood L, et al. Autism diagnostic observation schedule: a standardized observation of communicative and social-behavior. J Autism Dev Disord. 1989;19(2):185-212.

30. Wechsler D. The psychometric tradition: developing the wechsler adult intelligence scale. Contemp Educ Psychol. 1981;6(2):82-5.

31. Baron-Cohen S, Wheelwright S, Skinner R, Martin J, Clubley E. The AutismSpectrum Quotient (AQ): evidence from Asperger syndrome/highfunctioning autism, males and females, scientists and mathematicians. J Autism Dev Disord. 2001:31(1):5-17.

32. Foa EB, Huppert JD, Leiberg S, Langner R, Kichic R, Hajcak G, et al. The obsessive-compulsive inventory: development and validation of a short version. Psychol Assess. 2002;14(4):485-96.

33. Barkley RM, Murphy KR. Attention deficit hyperactivity disorder: a clinical workbook. 3rd ed. New York: Guilford; 2005.

34. Hamilton M. A rating scale for depression. J Neurol Neurosurg Psychiatry. 1960;23:56-62.

35. Hamilton M. The assessment of anxiety states by rating. Br J Med Psychol. 1959;32(1):50-5.

36. Salvadori C, Ward C, Defrance R, Hopkins R. The pharmacokinetics of the antidepressant tianeptine and its main metabolite in healthy humans: influence of alcohol coadministration. Fundam Clin Pharm. 1990;4(1):115-25.

37. Rubia K, Lee F, Cleare AJ, Tunstall N, Fu CH, Brammer M, et al. Tryptophan depletion reduces right inferior prefrontal activation during response inhibition in fast, event-related fMRI. Psychopharmacology. 2005;179(4):791-803.

38. Friedman NP, Miyake A. The relations among inhibition and interference control functions: a latent-variable analysis. J Exp Psychol Gen. 2004;133(1):101-35.

39. Corp. I. IBM SPSS Statistics for Windows. 2013; Version 22.0.

40. Brammer MJ, Bullmore ET, Simmons A, Williams SC, Grasby PM, Howard $\mathrm{RJ}$, et al. Generic brain activation mapping in functional magnetic resonance imaging: a nonparametric approach. Magn Reson Imaging. 1997;15(7):763-70.
41. Talairach JTP. Co-planar stereotaxic atlas of the human brain. New York: Thieme; 1988.

42. Corbett BA, Constantine LJ. Autism and attention deficit hyperactivity disorder: assessing attention and response control with the integrated visual and auditory continuous performance test. Child Neuropsychol J Norm Abnorm Dev Childh Adolesc. 2006;12(4-5):335-48.

43. Jeong JW, Chugani DC, Behen ME, Tiwari VN, Chugani HT. Altered white matter structure of the dentatorubrothalamic pathway in children with autistic spectrum disorders. Cerebellum. 2012;11(4):957-71.

44. Catani M, Jones DK, Daly E, Embiricos N, Deeley Q, Pugliese L, et al. Altered cerebellar feedback projections in Asperger syndrome. Neurolmage. 2008;41(4):1184-91.

45. Noonan SK, Haist F, Muller RA. Aberrant functional connectivity in autism: evidence from low-frequency BOLD signal fluctuations. Brain Res. 2009;1262:48-63.

46. Khan AJ, Nair A, Keown CL, Datko MC, Lincoln AJ, Muller RA. Cerebro-cerebellar resting-state functional connectivity in children and adolescents with autism spectrum disorder. Biol Psychiat. 2015;78(9):625-34.

47. Courchesne E, Campbell K, Solso S. Brain growth across the life span in autism: age-specific changes in anatomical pathology. Brain Res. 2011;1380:138-45.

48. Ecker C, Rocha-Rego V, Johnston P, Mourao-Miranda J, Marquand A, Daly $E M$, et al. Investigating the predictive value of whole-brain structural MR scans in autism: a pattern classification approach. Neurolmage. 2010:49(1):44-56.

49. Howes OD, Rogdaki M, Findon JL, Wichers RH, Charman T, King BH, et al. Autism spectrum disorder: consensus guidelines on assessment, treatment and research from the British Association for Psychopharmacology. J Psychopharmacol. 2018;32(1):3-29.

50. Lechin F, van der Dijs B, Hernandez G, Orozco B, Rodriguez S, Baez S. Acute effects of tianeptine on circulating neurotransmitters and cardiovascular parameters. Prog Neuropsychopharmacol Biol Psychiatry. 2006;30(2):214-22.

51. McEwen BS, Chattarji S, Diamond DM, Jay TM, Reagan LP, Svenningsson $\mathrm{P}$, et al. The neurobiological properties of tianeptine (Stablon): from monoamine hypothesis to glutamatergic modulation. Mol Psychiatry. 2010;15(3):237-49.

52. Samuels BA, Nautiyal KM, Kruegel AC, Levinstein MR, Magalong VM, Gassaway MM, et al. The behavioral effects of the antidepressant tianeptine require the mu-opioid receptor. Neuropsychopharmacology. 2017:42(10):2052-63.

53. Horder J, Lavender T, Mendez MA, O'Gorman R, Daly E, Craig MC, et al. Reduced subcortical glutamate/glutamine in adults with autism spectrum disorders: a [H-1]MRS study. Transl Psychiatry. 2014;4:e364

54. Pellissier LP, Gandia J, Laboute T, Becker JAJ, Le Merrer J. mu opioid receptor, social behaviour and autism spectrum disorder: reward matters. $\mathrm{Br}$ J Pharmacol. 2018;175(14):2750-69.

55. Chantiluke K, Barrett N, Giampietro V, Brammer M, Simmons A, Murphy $D G$, et al. Inverse effect of fluoxetine on medial prefrontal cortex activation during reward reversal in ADHD and autism. Cereb Cortex. 2015;25(7):1757-70.

\section{Publisher's Note}

Springer Nature remains neutral with regard to jurisdictional claims in published maps and institutional affiliations. 